\title{
LA IGNORANCIA SOCRÁTICA COMO VIRTUD EPISTÉMICA*
}

\author{
MARCELO D. BOERI \\ Pontificia Universidad Católica de Chile
}

\begin{abstract}
RESUMEN: En este artículo argumento que un tipo particular de ignorancia, de connotaciones «socráticas», es central para la adquisición de conocimiento dada la estructura epistémica de la sociedad. Sócrates advirtió la relevancia de un cierto tipo de ignorancia en la adquisición de conocimiento y fue consciente de la división del trabajo epistémico en la sociedad. Primero explico la manera en que entiendo la llamada «ignorancia socrática» (IS) como diferente de lo que llamo «ignorancia obstinada» (IO). Luego, discuto algunos vicios intelectuales (como la arrogancia epistémica) y la función que las virtudes intelectuales (la humildad epistémica y la autonomía) pueden tener respecto de la ignorancia obstinada como un correctivo que tiene alcances no sólo epistémicos, sino también morales. Este tipo de enfoque puede ser muy útil para explotar algunos supuestos de la epistemología social contemporánea, según los cuales la adquisición de conocimiento se hace a partir de los demás y con los demás.
\end{abstract}

PALABRAS CLAVE: Sócrates; Platón; ignorancia; conocimiento; virtud epistémica.

\section{The Socratic Ignorance as Epistemic Virtue}

ABSTRACT: In this article I argue that an especial kind of ignorance, of «Socratic» overtones, is central to the acquisition of knowledge given the epistemic structure of society. Socrates noted the relevance of a certain type of ignorance in the acquisition of knowledge and was aware of the epistemic division of labor in society. Firstly, I explain how I understand the so-called «Socratic Ignorance» (SI) as different from what I will call «stubborn ignorance» (SI). Then, I discuss some intellectual vices (such as epistemic arrogance) and the role that intellectual virtues (epistemic humility and autonomy) can have regarding stubborn ignorance as a corrective that has both epistemic and moral implications. This kind of approach can be very helpful for exploiting some assumptions of contemporary social epistemology, according to which the acquisition of knowledge is made from others and with others.

KEY WORDS: Socrates; Plato; Ignorance; Knowledge; Epistemic Virtue.

\section{SócRATES y LA EPISTEMOLOGÍA CONTEMPORÁNEA}

El Sócrates de Platón hizo célebre la tesis de la docta ignorantia, esto es, el enfoque según el cual la ignorancia puede contener algún aspecto propio de la sabiduría, una idea que encierra una especie de oxímoron incomprensible. Jenofonte atribuye a Sócrates la tesis de que el no conocerse a sí mismo ( $\tau$ ò

\footnotetext{
Presenté distintas versiones de este texto en la Universidad de la República (Uruguay, en el marco del IV Congreso de la Sociedad Filosófica del Uruguay, junio de 2018, organizado por el Prof. Carlos Caorsi) y en la Universidad Católica Argentina (como ponente en las «Jornadas de estudio sobre la epistemología platónica y su recepción», que tuvieron lugar en la Facultad de Filosofía y Letras de la Universidad Católica Argentina, organizadas por la Prof. Ivana Costa en el Instituto de Estudios Grecolatinos Prof. F. Nóvoa). Por sus preguntas y comentarios agradezco a los asistentes a esas reuniones y en especial a mis anfitriones (Carlos Caorsi e Ivana Costa). Algunos aspectos de este texto también los discutí con Leandro De Brasi (con quien estoy agradecido), en el marco de un proyecto conjunto. Este artículo es un resultado parcial del Proyecto Fondecyt 1200213 (Chile), del cual soy Investigador Responsable.
} 


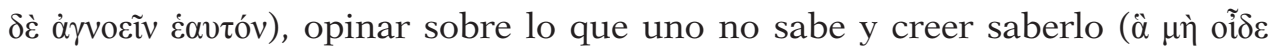

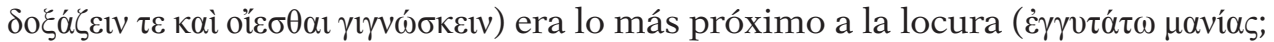
Recuerdos de Sócrates III 9, 6). En otro pasaje del mismo texto Jenofonte le hace decir a Sócrates que el "conócete a ti mismo" ( condición de cualquier otro saber: si uno no se conoce a sí mismo, difícilmente podría saber o conocer alguna otra cosa (Recuerdos de Sócrates IV 2, 24) ${ }^{1}$. La afirmación, atribuida a Sócrates por Platón, según la cual lo que en el mejor de los casos uno puede saber es que sabe que no sabe se repite con frecuencia, pero no es particularmente clara: se podría preguntar, por ejemplo, cómo es posible que la ignorancia suponga alguna forma de saber. Uno podría responder a esta pregunta citando una sección final del Teeteto (un diálogo ciertamente tardío que, sin embargo, tiene aspectos muy socráticos) en la que el personaje Sócrates afirma que, si después del largo, arduo y por momentos embrollado debate que se ha tenido acerca del saber o conocimiento ( $\dot{\varepsilon}\lrcorner \sigma \tau \eta \dot{\mu} \mu)$ uno intenta «quedar encinta» de otras cosas, estará lleno de cosas mejores gracias al examen que se ha llevado a cabo. En cambio, si uno queda estéril, será menos pesado y más amable para quienes se relacionan con uno mismo pues sensatamente

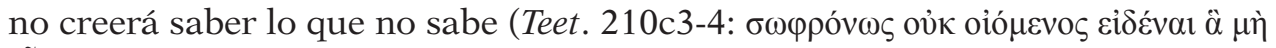
oĩota; cfr. también 187c, 196c, 199e).

Una corriente reciente de la epistemología contemporánea otorga especial relevancia a la ignorancia en el marco de una teoría epistemológica. Si uno ha leído algunos de los pasajes más significativos de los diálogos platónicos en los que la ignorancia socrática se presenta como el "vestíbulo del conocimiento», seguramente no se sorprenderá de este enfoque, aparentemente redescubierto por la filosofía contemporánea. Visto el problema desde una perspectiva ingenua, se puede pensar que la ignorancia no puede tener nada de atractivo, ya que prácticamente toda elección que se haga se la hace en el trasfondo de una ignorancia masiva sobre el propio pasado, el futuro, las circunstancias actuales, o sobre uno mismo ${ }^{2}$. Pero si se reflexiona sobre el tema, no es menos cierto que tiene o puede tener conciencia de todo esto, y si la tiene, lo sabe en algún sentido de saber o conocer.

Según el «enfoque estándar» de la epistemología contemporánea, la ignorancia puede entenderse como falta o ausencia de conocimiento. Algunos argumentan que el rol de la ignorancia ha sido descuidado en la epistemología y quieren mostrar que tiene o puede tener una función importante en las teorías que intentan explicar qué es el conocimiento, cuáles son sus condiciones mínimas, qué elementos son más o menos valiosos en el conocimiento, por qué es relevante saber qué es el conocimiento, y ello no sólo en el sentido de

1 Para el «conócete a ti mismo» en Platón cfr. Cármides 165a; Alcibíades I 124a, 129a; Protágoras 343a-b; Filebo 48c-d; Leyes 923a; Carta VII 341b-c. Para Platón he empleado el texto griego editado por BuRnet, J., Platonis Opera (Recognovit brevique admnotatione critica instruxit Ioannes Burnet), Tomvs I-IV, Oxford, Oxford Classical Texts 1976 (reprinted).

2 Sobre este interesante detalle cfr. Zimmerman, M. J., Living with Uncertainty. The Moral Significance of Ignorance, Cambridge University Press, Cambridge, 2008, p. ix. 
ser capaz de dar una definición correcta de conocimiento, sino también en el sentido de una vida práctica efectiva, es decir, una vida vivible para un ser humano.

Argumentaré que un tipo particular de ignorancia, de connotaciones «socráticas», es central para la adquisición de conocimiento dada la estructura epistémica de la sociedad. Sostendré que Sócrates advirtió la relevancia de un cierto tipo de ignorancia en la adquisición de conocimiento y que fue consciente de la división del trabajo cognitivo que encontramos en nuestra sociedad. Dicho de otro modo, lo que uno efectivamente sabe es muy poco, no es posible saberlo todo, y si uno cree que lo sabe todo (o todo lo que se refiere de modo directo a su vida) puede estar experimentando una ilusión de conocimiento. Pero si uno no puede saber todo (como, de hecho, no puede), debe recurrir a los demás. Esto último, desde luego, plantea desafíos formidables: por ejemplo, si uno siempre es un lego en un cierto dominio cognitivo, ¿qué criterio puede tener para saber a quién recurrir? ¿Por qué seguir lo que a uno le dice el experto (o supuesto experto) $1\left(\mathrm{E}^{1}\right)$ y no lo que le dice el experto $2\left(\mathrm{E}^{2}\right)$ ?

En lo que sigue explicaré primero la manera en que entiendo la llamada «ignorancia socrática» (IS) como un tipo de ignorancia diferente de lo que llamaré «ignorancia obstinada» (IO). También voy a discutir brevemente algunos vicios intelectuales (uno de los más representativos es la arrogancia epistémica) y la función que las virtudes intelectuales (como la humildad epistémica y la autonomía) pueden tener respecto de la ignorancia obstinada o contumaz como un correctivo que tiene alcances no sólo epistémicos, sino también morales. Creo que este tipo de enfoque puede ser muy fértil para leer algunos textos socrático-platónicos y para mostrar que, aunque Platón no tenía ciencia empírica de la mente ni algunos recursos conceptuales con los que cuenta la filosofía contemporánea, puede establecerse un diálogo razonablemente útil entre el pensamiento platónico y el contemporáneo. También espero mostrar que para explotar algunos supuestos de la epistemología social contemporánea, según los cuales la adquisición de conocimiento se hace a partir de los demás y con los demás, los textos platónicos pueden tener un rol particularmente relevante.

Voy a discutir algunas tesis y argumentos atribuidos al filósofo Sócrates, es decir, el personaje central de algunos diálogos de Platón ${ }^{3}$. Sócrates usa las

\footnotetext{
3 «Sócrates» es una brillante construcción literaria y filosófica de Platón, un gran filósofo que no se limitó a describir la figura humana e intelectual de su maestro. Es posible que el Sócrates platónico contenga algunas características genuinas del Sócrates histórico, pero podemos sospechar que también posee rasgos que proceden del filósofo Platón (para el «problema de Sócrates» cfr. Waterfield, R., «The Quest for the Historical Socrates», en: Bussanich, J., Smith, N. D. (eds.), The Bloomsbury Companion to Socrates, London-New Delhi-New York-Sydney, 2013, pp. 1-19 y, más recientemente, VALLEJo Campos, A., «Sócrates» en: Vallejo Campos, A. y Vigo A. G., Filósofos griegos: de los sofistas a Aristóteles, Eunsa, Pamplona, 2017, pp. 93-104). La representación que Platón habitualmente hace de Sócrates es seguramente la que ha dado lugar a la figura de significación filosófica perenne (cfr. TAYLOR, C. C. W., "Socrates», en: TaYlor, C. C. W., Pleasure, Mind, and Soul. Selected Papers in Ancient
} 
nociones de conocimiento e ignorancia de maneras que no siempre ni en todos los casos son coherentes ${ }^{4}$; de cualquier modo, me voy a referir a algunas tesis y argumentos que, si bien no parecen coincidir en todos sus detalles con otros diálogos, de todos modos, es posible encontrar un hilo conductor común que describe un pensamiento relativamente coherente.

Como ya he señalado, el tema de este artículo está inspirado por la corriente de la epistemología contemporánea que confiere especial relevancia a la ignorancia en el marco de una teoría epistemológica. La epistemología como disciplina filosófica se entiende generalmente como una teoría que pretende explicar, entre otras cosas, la naturaleza, alcance y valor del conocimiento,

Philosophy, Clarendon Press, Oxford, 2008, p. 295; 301-304). En las últimas décadas, sin embargo, los estudiosos de Sócrates han revitalizado la importancia que también tiene la figura del Sócrates de Jenofonte (una enjundiosa comparación entre el Sócrates platónico y el jenofonteo puede verse en Renaud, F., "Les Mémorables de Xénophon et le Gorgias de Platon: étude comparative», en: Narcy, M., Tordesillas, A. (eds.), Xénophon et Socrates, Vrin, Paris, 2008, pp. 161-180. Para la discusión de este espinoso tema cfr. KAHN, C., Plato and the Socratic Dialogue. The Philosophical Use of a Literary Form, Cambridge, Cambridge University Press, 1996, pp. 71-95 y, Dorion, L.-A., Que sais-je? Socrate, Presses Universitaires de France, Paris, 2011, caps. II-V (2e édit.), quien suministra un detallado análisis de los «variados Sócrates» (el de Aristófanes, el de Platón, el de Jenofonte y el de Aristóteles). Es interesante observar que el Sócrates de Jenofonte nunca declara ser ignorante sobre los asuntos que trata (particularmente los asuntos éticos). El Sócrates platónico, en cambio, explícitamente declara que él es ignorante acerca de lo que se relaciona con las virtudes (ver Cármides 165b-c).

4 Algunas de las opiniones que prevalecen sobre el significado de la expresión «ignorancia socrática» se proponen explicar dicha ignorancia en relación con su ironía, a saber, el «disimulo de Sócrates de su ignorancia» (para el significado de eipoveía como "disimulo», cfr. Cicerón, Academica II 15, quien sugiere que debe entenderse como una especie de dissimulatio, pues Sócrates decía algo diferente de lo que en realidad pensaba: cum aliud diceret atque sentiret. Véase también Aulo Gelio, Noches Áticas XVIII 4, 1). A diferencia de la certeza de conocimiento exhibida por sus interlocutores, Sócrates se posiciona a sí mismo, por un lado, como un «admirador entusiasta» de la supuesta sabiduría que tienen y, por el otro, está preocupado por su propia ignorancia, que es absoluta: es consciente de que no sabe

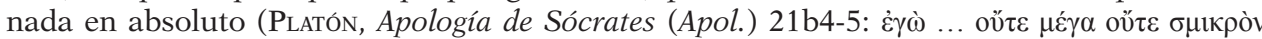

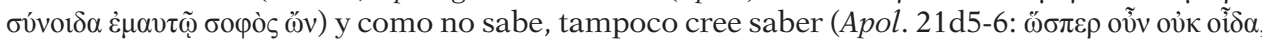

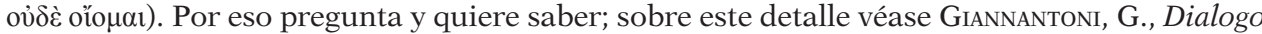
socratico e nascita della dialettica nella filosofía di Platone (Edizione postuma a cura di Bruno Centrone), Bibliopolis, Napoli, 2005, p. 124 (ver también p. 56, n. 43). Otros estudiosos, al adoptar un punto de vista unitario en lugar de uno desarrollista, intentan encontrar una solución al "problema de la ignorancia socrática» y argumentan que hay cierta evidencia textual en la que Sócrates hace «una reivindicación explícita del conocimiento»y, por lo tanto, intentan resolver el problema de la ignorancia socrática al sostener que las negaciones de Sócrates sobre el conocimiento son irónicas y que deben ser rechazadas (cfr. MACPARTLAND, K., «Socratic Ignorance and Types of Knowledge», en: Bussanich, J. y Sмith, N. D. (eds.), The Bloomsbury Companion to Socrates, Bloomsbury, London-New Delhi-New York-Sydney, 2013, pp. 95-96). Mi opinión sobre el problema de la ignorancia socrática es mucho más simple y deflacionaria: Sócrates reconoce que es ignorante, de modo que tal reconocimiento puede entenderse como una especie de virtud epistémica (i.e., "humildad epistémica»). De este modo, hago que Sócrates entre en debate con algunos epistemólogos contemporáneos que consideran la ignorancia como un ingrediente positivo en la búsqueda del conocimiento. 
pero deja otros bienes epistémicos relativamente inexplorados a menos que estén directamente relacionados con el conocimiento (por ejemplo, algún tipo de justificación epistémica que se considera necesaria para ello). Tanto en las definiciones lexicográficas que encontramos en los diccionarios ordinarios como en las que se proporcionan en los diccionarios de filosofía, la ignorancia es lo opuesto al conocimiento, por lo que ella a veces meramente se entiende como falta o ausencia de conocimiento (ésta es la supuesta visión estándar sobre qué es ignorancia) $)^{5}$. Es en este sentido que se puede argumentar que el conocimiento y la ignorancia son términos complementarios: el sujeto $S$ ignora $p$ si y solo si $S$ no conoce $p^{6}$. Obviamente, decir que la ignorancia es falta o ausencia de conocimiento es una caracterización apropiada de lo que es ignorancia, pero no es muy informativa ni esclarecedora: por ejemplo, no dice mucho acerca de su papel, si es que lo tiene, en el dominio epistemológico. Tal comprensión de la ignorancia se puede tomar como una perogrullada que no agrega demasiado; por eso, se argumenta, el tema de la ignorancia ha sido descuidado en el tratamiento técnico-filosófico de la epistemología ${ }^{7}$ y lo que estos enfoques recientes en los debates epistemológicos contemporáneos quieren hacer, es mostrar el papel (central, según algunos filósofos) de la ignorancia en teorías que intentan explicar qué es el conocimiento.

Me interesa mostrar que un tipo particular de ignorancia, de connotaciones «socráticas», es esencial para la adquisición de conocimiento dada la estructura epistémica de la sociedad ${ }^{8}$. No pretendo sugerir que Sócrates ya había notado todas las dificultades asociadas al papel (a veces positivo) de la ignorancia en un proyecto epistemológico, o que su proyecto fuera un "proyecto epistemológico» en el sentido contemporáneo de la expresión. Pero está claro que advirtió la relevancia de cierto tipo de ignorancia en la adquisición de conocimiento y que era consciente de la división del trabajo epistémico y cognitivo que podemos encontrar en nuestra sociedad. Para mostrarlo explicaré cómo entiendo la llamada «ignorancia socrática» (IS), en oposición a la «ignorancia obstinada» (IO), y la función que se puede atribuir a la ignorancia y las virtudes intelectuales, las que pueden entenderse como «correctivos» de dicha ignorancia.

5 Cfr. Le Morvan, P. y Peels, R., «The Nature of Ignorance: Two Views», en: Peels, R., Blaauw, M. (eds.), The Epistemic Dimensions of Ignorance, Cambridge University Press, Cambridge: 2016, p. 12-32. El otro punto de vista alternativo importante (que la ignorancia es la ausencia de creencia verdadera) puede entenderse como la falta de conocimiento débil, a diferencia del conocimiento fuerte (que requiere más que la mera creencia verdadera), como lo hace el punto de vista estándar. Véase Goldman, A., Knowledge in a Social World, Oxford University Press, Oxford, 1999, p. 23; 324.

6 Goldman, A. y Olsson, E. J., «Reliabilism and the Value of Knowledge», en: Haddock, A., Millar, A. y Pritchard, D. (eds.), Epistemic Value, Oxford University Press, Oxford, p. 19-20.

7 Cfr. Peels, R. y Blaauw, M., "Introduction», en: Peels, R. y Blaauw, M. (eds.), The Epistemic Dimensions of Ignorance, Cambridge University Press, Cambridge, 2016, p. 1-2.

8 De un modo cuasi-socrático Pritchard señala que, aunque la ignorancia es una posición epistémica negativa, de ello no se sigue que sea una posición epistémica disvaliosa: (cfr. Pritchard, D., «Ignorance and Epistemic Value», en: Peels, R y BlaAuw, M. (eds.), op. cit., p. 132. 
Voy a comenzar por discutir el principio socrático, según el cual la peor ignorancia es creer que uno sabe lo que realmente no sabe, una tesis que aparece por primera vez en los «diálogos socráticos» (Apol. 21d) y que permanece intacta hasta los diálogos de vejez (Teeteto 210c; Sofista 229c). Luego consideraré la estructura epistémica de la sociedad, con su división del trabajo cognitivo, y discutiré brevemente el problema de la ilusión de conocimiento, la que, probablemente, el ignorante sufre debido a esta estructura epistémica, y que resulta en lo que he llamado IO. En la sección 3 trataré el papel de la IS en la adquisición de conocimiento y presentaré las virtudes intelectuales de la humildad y la autonomía (ambas, sostengo, en parte adelantadas y conceptualizadas por Sócrates), las que se requieren para explotar la estructura epistémica antes mencionada y para luchar contra la IO. En el apartado final me refiero brevemente a la relevancia de la IS y las virtudes epistémicas asociadas a ella para la deliberación política.

\section{SócRATES, LA CONFIANZA EN LAS PROPIAS CREENCIAS Y LA ILUSIÓN DE CONOCIMIENTO}

Sócrates dice que es consciente de que no es sabio en absoluto (Apol. 21b4$5)^{9}$; si no sabe nada, debe recurrir a los supuestos expertos ( $u$ a otra autoridad epistémica $)^{10}$. Eso es precisamente lo que hace Sócrates en la Apología: dado que

9 Es cierto que, aunque Sócrates declara que no es sabio, tiene y afirmas varias opiniones sobre lo que es la virtud, como si la conociera; por ejemplo, que la virtud es una especie de sabiduría (Apol. 29e). También afirma positivamente que sabe (oĩ $\alpha$ ) que es malo y vergonzoso cometer injusticia y desobedecer al que es mejor, ya sea que se trate de un dios o de un hombre (Apol. 29b6-7). Además, en Apol. 40a4-6 Sócrates admite que «el habitual [poder] profético de

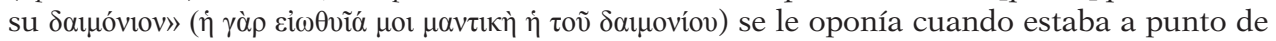
hacer algo malo, implicando de esta manera que, hasta cierto punto, era consciente de lo que

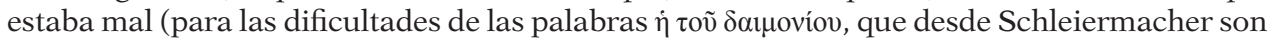
entendidas como una glosa, un agregado innecesario, por los editores, cfr. Vigo, A. G., Platón, Apología de Sócrates, Ed. Colihue, Buenos Aires, 2018, p. 161). En el Eutidemo está seguro de que los buenos no son injustos (296e8). Es claro que el conocimiento que es relevante para Sócrates es el relacionado con los asuntos éticos; de todas maneras, comienza su investigación examinando a aquellos que declaran tener un conocimiento, sin importar que ese conocimiento sea un conocimiento «técnico» (como la política, la artesanía, la poesía, etc.). La pregunta podría ser cómo puede existir la profesión socrática de ignorancia junto con su confianza acerca de lo que es virtud y lo que es bueno y malo.

10 Un individuo califica como genuino experto (i.e., no como supuesto experto) si dispone de un cuerpo extenso e integrado de conocimientos especializados, relacionados tanto con los hechos como con las metodologías relevantes. Una autoridad epistémica sabe que el receptor del testimonio intenta, pero no necesita ser un experto (cfr. ZAGZEBSKI, L., Epistemic Authority: A Theory of Trust, Authority, and Autonomy in Belief, Oxford University Press, New York, 2012, p. 109). Por ejemplo, alguien podría ser una autoridad epistémica para uno en la prosa borgeana simplemente por haber asistido a una charla relevante sobre el tema. Un experto, en cambio, si efectivamente es una autoridad epistémica genuina, debe poseer una cantidad y calidad significativamente mayor de conocimiento sobre el tema relevante que la mayoría de la gente. Además, debe poseer la capacidad de formar creencias verdaderas a las 
se considera ignorante, busca personas que supuestamente tienen una pericia epistémica relevante. Si es ignorante en un campo de especialización dado y advierte que está en desacuerdo con un experto en esa área, normalmente debe adoptar el punto de vista del experto. Pero ¿qué evidencia puede tener uno, siendo un lego, de que alguien, cuando declara ser un experto, realmente sabe lo que dice saber? El Sócrates de los primeros diálogos de Platón advierte este problema (que en la discusión epistemológica contemporánea no es menor) ${ }^{11}$ : en el Cármides, por ejemplo, (y como conclusión de un largo y sofisticado argumento), Sócrates sostiene que uno «no puede distinguir al que finge ser un médico, a pesar de que no lo es, de quien realmente lo es, ni de ninguno que tenga conocimiento de quienes no lo tienen» (170d-e; mi traducción). La dificultad que todos tenemos como legos es cómo evaluar a los supuestos expertos (éste es el problema, señalado antes — cfr. supra p. 105—, respecto de cómo confiar más en lo que dice $\mathrm{E}^{1} \mathrm{y}$ no en lo que dice $\mathrm{E}^{2}$ ).

Pero la confianza en la pericia de los demás es una característica omnipresente de la vida moderna (una característica que sin duda también estaba presente en la época de Platón, como se muestra en varios de sus diálogos y en las conversaciones que el personaje Sócrates mantiene con los supuestos sabios o expertos) y el problema recién mencionado es, por supuesto, más manejable que tener que aprender un nuevo dominio del conocimiento. En efecto, uno no puede saber todo, pero tampoco puede ponerse a la tarea de aprender todo con la intención, siempre poco prometedora, de poder saber todo. No sólo eso, sino que, dado que nadie puede saber todo y el hecho de que vivimos en una sociedad de conocimientos hiper-especializados, que distribuye el trabajo epistémico entre diferentes personas, es más deseable procurar aprender las habilidades para discriminar entre las fuentes buenas y malas de conocimiento de los testimonios (así como los contenidos). Si uno necesita saber qué le está sucediendo a su hombro dolorido, lo que debe buscar es el testimonio del médico, y no de cualquier médico, sino de un traumatólogo, que es el médico especializado en el tratamiento de ese tipo de dolencias (es decir, el testimonio del experto en el área de su competencia, que es un área obviamente especializada), y podrá saber eso y lo que puede hacer para eliminar los síntomas no deseados. Por tanto, uno no necesita saber todo, ni puede hacerlo: cuando me duele mi hombro o cuando quiero entender la teoría de Georg Cantor sobre los múltiples infinitos no puedo pretender convertirme en traumatólogo o en matemático. Cada uno de nosotros tiene un papel que desempeñar en la división del trabajo epistémico y puede, al menos en principio, confiar en los demás respecto del conocimiento especializado que no posee y que es provisto por los expertos.

nuevas preguntas que puedan plantearse dentro del tema (cf. Goldman, A., «Experts: Which Ones Should You Trust?», Philosophy and Phenomenological Research vol. 63, N 1 (2001), p. 91). Si eso es así, el experto es claramente el superior epistémico de un lego (en el área de especialización correspondiente).

11 Cfr. Goldman, op. cit., p. 85-86; Coady, C. A., What to Believe Now: applying epistemology to contemporary issues, Wiley-Blackwell, Chichester, 2012, chap. 2. 
La estructura epistémica de la sociedad, con su división del trabajo cognitivo, no requiere que enseñemos todo a todos y que enseñemos a las personas solamente a pensar por sí mismas ${ }^{12}$; por ejemplo, si soy un filósofo experto en fenomenología alemana y un colega procedente de la tradición analítica quiere saber el sentido en que los fenomenólogos alemanes de la primera mitad del s. XX usan la expresión «intencionalidad», no es necesario que mi colega analítico conozca absolutamente todos los detalles de la fenomenología alemana y que, por ende, se convierta en un experto en esa tradición filosófica. Basta con describir de una manera más o menos precisa qué se entiende en la fenomenología alemana por «intencionalidad», de modo que sea capaz de hacer las conexiones pertinentes de esa noción con lo que los filósofos analíticos entienden por ese tecnicismo. Visto el problema desde esta perspectiva, es claro que la tarea de adquisición de conocimiento es compartida. Como es obvio, los expertos están ahí para «ser explotados» y las personas deben aprender a hacerlo para garantizar una comunidad epistémica saludable. Ignorar el consejo de un experto no es una opción realista, al menos dado el hecho de que nadie puede saber todo en una sociedad hiper-especializada; esto implica que todo el mundo es ignorante en muchos dominios diferentes (o tiene un conocimiento muy limitado en comparación con los expertos). Esta ignorancia (parcial) no es negativa dada esta división del trabajo epistémico; además, es importante reconocer que los demás pueden saber más que uno mismo sobre ciertas cosas, a saber, los dominios en los que ellos se especializan y uno no. Es decir, debemos reconocer nuestra ignorancia y ser capaces de depender epistémicamente de los demás en ciertas circunstancias.

Seguramente las connotaciones socráticas de este tipo de razonamiento no deberían sorprendernos: en la Apología de Sócrates, el personaje Sócrates argumenta que su conocimiento es un "conocimiento humano» en la medida en que lo único que en rigor sabe es que no sabe o que no cree saber lo que en realidad no sabe (una descripción de su conocimiento que describe la virtud epistémica de la humildad). Es una sabiduría o conocimiento que se limita a la conciencia de los límites del propio saber ${ }^{13}$. Sócrates no habla de «sociedad cognitivamente hiper-especializada», pero sin duda es completamente consciente de que hay dominios cognitivos específicos y de que el hecho de poseer un saber en un área profesional no habilita a quien posee dicho conocimiento

12 Algunos filósofos y psicólogos cognitivos han sugerido recientemente que la razón evolucionó para funcionar en una interacción que va de un lado a otro (interactive backand-forth; cfr. Mercier, H. y SPERberg, D., The Enigma of Reason, Harvard University Press, Cambridge, Massachusetts, 2017, pp. 283-284). Si lo que argumentan Mercier-Sperberg es cierto, estas divisiones del trabajo cognitivo no sólo son facetas muy importantes y naturales de las personas como individuos, sino también de la estructura de la sociedad.

13 Para este importante detalle (que atraviesa prácticamente la totalidad de la obra platónica) cfr. Apol. 20d-e; 23a-b; 29b, VIGo, A. G., op.cit. p. xix, y las notas al texto incluidas en pp.148-150. 
a conocer también otras áreas en los cuales es un lego ${ }^{14}$. Sin embargo, el ignorante (entendido como aquel que, a diferencia del experto, carece de un conocimiento experto en algún dominio profesional) normalmente carece de la capacidad requerida para reconocer su ignorancia. Esto puede generar en los ignorantes una ilusión en cuanto a la cantidad (y calidad) de conocimiento que poseen, lo que actualmente se denomina «ilusión de conocimiento ${ }^{15}$. Peor aún, el ignorante también es «bendecido» por el exceso de confianza; desde luego que todos padecemos un sesgo de exceso de confianza, por el cual tenemos la impresión de estar mejor informados que lo que en realidad estamos, y tener más confianza de lo que deberíamos en nuestros puntos de vista. Éste es un aspecto del problema de la ignorancia y del efecto devastador que ejerce en quien la posee ampliamente reconocido por los psicólogos cognitivos y filósofos contemporáneos, pero que ya fue advertido por Platón. En Leyes IX (863c) argumenta de la siguiente manera: la ignorancia es la causa de los errores y debería distinguirse así: (a) una parte de ella es simple y causa de errores leves, la otra $(b)$ es doble, porque una persona puede ser ignorante y su ignorancia es sostenida no sólo por su propia ignorancia, sino también por «la apariencia

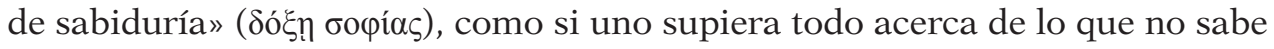
nada. Esto es, la «ignorancia doble» es la que no sólo es ignorancia, sino que además está respaldada por una apariencia de sabiduría (que presupone creer que uno posee un saber, aunque en rigor no lo posee) y generar de ese modo la imposibilidad de ser capaz de reconocer el propio error y producir una ilusión de conocimiento (cfr. también Leyes 732a-b; 886b7-8).

Ahora bien, si atendemos a esta tendencia general a sobreestimar el peso epistémico de nuestras propias creencias, cabe señalar el llamado «efecto KrugerDunning», según el cual cuanto más ignorante (y, en general, incompetente) es una

14 Cfr. Platón, Apol. 22d-e, donde Sócrates reconoce que los artesanos poseen un cierto saber, pero les reprocha que cometen el mismo error que los poetas: "por realizar bien su arte, cada uno de ellos estimaba que también era extremadamente competente o sabio

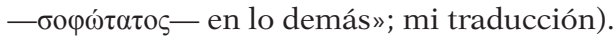

15 Cfr. Sloman, S. y Ferniach, P. The Knowledge Illusion: Why we never think alone, Macmillan, London, 2017, quienes afirman que la ilusión de conocimiento «significa que no revisamos nuestra comprensión frecuentemente o con la suficiente profundidad. Esta es una receta para el pensamiento anticientífico» (p. 247: véase también pp. 313, 375 et passim). De nuevo, el carácter "cuasi-socrático» de este tipo de afirmación radica en el hecho de que, como pensaba Sócrates, quien está instalado en una ilusión de conocimiento se encuentra demasiado confiado acerca de «lo mucho que sabe». Esto puede tener algún beneficio (por ejemplo, de la arrogancia — que sólo puede describirse como ilusoria en ese momentoJohn F. Kennedy predijo que un americano aterrizaría a salvo en la luna a fines de los 60'). Pero la ilusión de conocimiento (y la arrogancia ilusoria que le es inherente) también puede tener efectos catastróficos (como la de Robert Scott en su expedición al Polo Sur; Scott se negó a usar perros porque «sabía lo que era mejor». Todos sus hombres y los animales que los acompañaban perecieron). Sócrates no estaría del lado de los presuntos beneficios de la ilusión de conocimiento, sino del de las catástrofes prácticas (y morales) que puede generar. 
persona, se siente más segura de que no es realmente ignorante ${ }^{16}$. El argumento de Kruger y Dunning puede reconstruirse así: (i) las personas por lo general son particularmente incompetentes para evaluarse a sí mismas y a sus habilidades; (ii) de donde en general se sigue que la persona promedio tiende a creer que está por encima del promedio, lo cual debe hacernos pensar que (iii) si nos focalizamos en los déficits metacognitivos de los que carecen de habilidades (the unskilled), es posible explicar esta tendencia general hacia autoevaluaciones (seguramente positivas) que son exageradas. Finalmente, (iv) dado que las personas generalmente eligen lo que creen que es la opción más razonable y óptima, si uno no reconoce que ha tenido un desempeño deficiente o pobre en una actividad determinada que exigía ciertas competencias cognitivas, terminará creyendo que su desempeño ha sido correcto. Por tanto, (v) los incompetentes tenderán a sobreestimar groseramente sus capacidades. Si es así, los ignorantes no sólo llegan a conclusiones erróneas (y, por ende, toman decisiones desafortunadas), sino que su ignorancia los priva de la capacidad de darse cuenta de su error y esto, a su vez, les permite crecer en cuanto a su propia confianza.

Dado lo anterior, el peor enemigo del conocimiento no es la ignorancia, sino la ilusión de conocimiento (es decir, creer erróneamente que uno posee un conocimiento). Para los seres humanos que vivimos en comunidades de conocimiento hiper-especializadas, la ignorancia es inevitable. Pero, dada la división del trabajo epistémico, esta ignorancia no es necesariamente algo malo. Entonces, puesto que uno no es ignorante acerca de su ignorancia con respecto a algún dominio (es decir, uno no sufre de una ilusión de conocimiento en un dominio dado, i.e., el dominio en el que es un experto) y acerca de quienes nos suministran conocimiento, la ignorancia (sobre algún dominio) no es el peor estado en el que uno puede encontrarse. Si uno padece una ilusión de conocimiento, dado que tal ilusión lo disuade a uno mismo de explotar la estructura epistémica societaria, uno se ve impedido de superar la ignorancia relevante. Vamos a ver enseguida que la IS (Ignorancia Socrática), entendida como la conciencia de la propia ignorancia en un dominio cognitivo dado, permite que dicha ignorancia sobre algún dominio no sea maligna, mientras que la IO (Ignorancia Obstinada), entendida como el resultado de una ilusión de conocimiento dentro de algún dominio, hace que la ignorancia sí lo sea.

\section{SóCRATES, SU IGNORANCIA, SU SABER Y POR QUÉ «LA INVESTIGACIÓN ES CONJUNTA»}

Lo sigue es una historia bien conocida, aunque espero que mi análisis pueda ser útil para enfatizar algunos aspectos que no siempre se destacan en la forma en que me gustaría presentar el tema. Cuando uno nota que otro es «más sabio»

16 Cfr. Kruger, J. y Dunning, D., «Unskilled and Unaware of It: How Difficulties in Recognizing One's Own Incompetence Lead to Inflated Self-Assessment» Psychology, 2009, 1, pp. 30-46 (especialmente pp. 31-32 y 37). 
o «más competente» que uno mismo en un área específica de conocimiento, y cuando es consciente de que uno está en desacuerdo con esa persona, uno normalmente tiende a aceptar su testimonio: si mi médico me dice que mi estilo de vida es perjudicial para mí (bebo y fumo excesivamente, no hago suficiente ejercicio y no me ocupo de tener una dieta balanceada), y si reconozco que mi médico sabe más que yo sobre mi salud, debo aceptar su testimonio ${ }^{17}$. Aceptar el testimonio del médico significa reconocer que el contenido de lo que me dijo luego de examinarme es cierto. Pero el Sócrates de algunos diálogos platónicos muestra con cierta frecuencia que las personas pueden ser obstinadamente resistentes a cambiar de idea, en especial cuando alguien les muestra que están o pueden estar en el error (parece que esto es algo particularmente poderoso en la psique humana, algo que Sócrates percibió con toda claridad). Hay un pasaje platónico muy claro al respecto que describe muy bien este hecho; es un texto que aparece en un diálogo tardío de Platón pero que, como el Teeteto, tiene ciertas connotaciones «socráticas». El personaje Filebo dice en el diálogo homónimo:

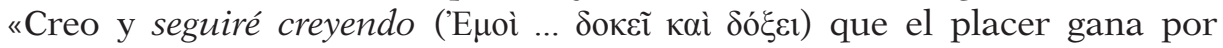
completo. ¡En cuanto a ti Protarco, tú sabrás!» (Filebo 12a7-8; mi traducción).

Este tipo de actitud recalcitrante justifica que Platón decida dejar al personaje Filebo fuera del debate. Obviamente, el personaje Filebo es una construcción literaria de Platón cuya intención es quizás describir el tipo de persona que prefiere conservar dogmáticamente sus creencias sin siquiera evaluar la posibilidad de presentarlas al escrutinio de la discusión dialógica ${ }^{18}$. La actitud de persistir en la creencia que uno tiene y el desinterés por someterla al examen revela una posición muy poco filosófica por parte de Filebo; es una actitud epistémicamente arrogante que muestra la falta de sensibilidad hacia la propia falibilidad y las deficiencias cognitivas. Pero la gente en general, como el personaje Filebo del diálogo homónimo ${ }^{19}$, cree casi todo lo que cree porque

17 Al menos debo hacerlo en un sentido teórico, aun cuando, de hecho, no cambie mi estilo de vida. Para un enfoque similar en Platón, pero en un contexto diferente, cfr. Teeteto $178 \mathrm{c}-\mathrm{d}$, donde trata de mostrar que el criterio del lego no puede ser equivalente al del experto (en su ejemplo, el experto es un médico). Su sugerencia implícita es que, si uno está enfermo, debe llamar a un médico (y, de hecho, es lo que uno habitualmente hace), no a una persona que no sabe nada de medicina.

18 O, como sugiere Gadamer, la actitud de Filebo muestra que renuncia a confrontar su punto de vista haciendo uso de la argumentación lógica, lo que siempre presupone recibir críticas y ser potencialmente refutable (GADAMER, H.-G., Die Idee des Guten zwischen Plato und Aristoteles, en: Gadamer, H.-G., Gesammelte Werke 7. Griechische Philosophie III, J. C. B. Mohr (Paul Siebeck) Tübingen, 1999, p. 187). Sobre la relevancia que tiene el método dialógico (entendido como "método de refutación») ver Natali, C. "Due dissertazioni scritte in fretta. Gadamer e Davidson sul Filebo di Platone», Méthexis XX, 2007, pp. 139-140 y Davidson, D., «Dialectic and Dialogue», en: Davidson, D., Truth, Language, and History, Oxford University Press, Oxford, 2005, pp. 252-254.

19 Sócrates sin duda fue consciente de que los seres humanos a menudo no estamos dispuestos a deshacernos de nuestras propias creencias. Como a menudo dice o sugiere, la gente cree lo que cree porque piensa que es verdad: de hecho, nadie cree que lo cree es falso (cfr. Cármides 166d-167a, pero también Teeteto 171a6-7; b1-5; 200a3 y Sofista 228c-d). Para 
le parece verdadero. Dicho de otro modo, algo que a uno le parece verdadero es casi siempre la causa inmediata (o próxima) para formar creencias (cualquiera que sea la causa distante o no próxima); pero nuestras creencias tienen diferentes causas distantes, no todas ellas son epistémicamente correctas y, por lo general, esas causas distantes son las que menos se tienen en cuenta a la hora de formar las propias creencias o de evaluar las que uno ya tiene. Es decir, si uno supone que el objetivo epistémico primario de la propia creencia es la verdad y que la (buena) evidencia de alguna creencia hace que ésta tenga más probabilidad de ser verdadera, las causas no evidentes de la creencia, como los prejuicios y los factores emocionales (como las expresiones de deseo) no cuentan como causas distantes epistémicamente apropiadas. Estos factores no evidentes o no probatorios no aumentan la probabilidad de que la creencia sea verdadera, pero la mayoría de las creencias (es decir, las que uno tiene por ciertas) parecen ser verdaderas para uno, independientemente de su causa distante. Por lo tanto, el hecho de que a uno le parezcan correctas no puede poner fin a los desafíos que surgen en su contra, como Filebo (debido a su arrogancia epistémica) podría pensar.

Para Platón, por el contrario, la actitud intelectual sana y el compromiso con la discusión dialógica presuponen la posibilidad de revisar las propias creencias y, si es necesario, modificarlas. Uno puede entender entonces que el Sócrates platónico promueva la virtud intelectual de la humildad epistémica, una virtud que se propone reducir la arrogancia epistémica al promover una actitud "escéptica» (en el sentido de una actitud investigativa). Esto puede ocurrir gracias al reconocimiento de la propia falibilidad y a la limitación de conocimiento, así como a una disposición a cambiar y modificar lo que uno cree gracias a las opiniones y a las razones de los demás. Más aún, según el Sócrates de Platón, "la investigación es compartida» ${ }^{20}$ y la refutación no debe entenderse como una afrenta, sino como una especie de correctivo del error, por así decir. En realidad, es lo que garantiza que uno sea capaz de revisar su propia opinión y, si es necesario, de corregirla ${ }^{21}$.

Sócrates, entonces, también parece sugerirnos que aprendamos a explotar cierta división del trabajo cognitivo, ya que juntos, al presentar diferentes razones a favor y en contra de una tesis, argumentamos en nuestra búsqueda conjunta de la verdad. Este tipo de división del trabajo cognitivo se ve en todo

un enfoque similar en Davidson, probablemente inspirado por Platón, ver DAvidSON, D., «First Person Authority», en: Davidson, D., Subjective, Intersubjective, Objective, Clarendon Press, Oxford, 2001, p. 4-6.

20 Para la discusión de la tesis de que la investigación debe ser conjunta o compartida cfr. Gill, C., "Afterword: Dialectic and the Dialogue Form in Late Plato», en: Gill, C. y McCABE, M. M. (eds.), Form and Argument in Late Plato, Clarendon Press, Oxford, 2004, p. 286, n.7; p. 289, n.13 y GILL, C., «Form and outcome of arguments in Plato's Gorgias», en: ERLER, M. y Brisson, L. (eds.), Gorgias-Menon. Selected papers from the Seventh Symposium Platonicum, Academia Verlag, Sankt Augustin, 2007, p. 62-65.

21 Cfr. Platón, Gorgias 458a2-b1 (que discuto brevemente abajo). Véase también Eutidemo 295a y Sofista 230b-e. 
tipo de deliberaciones, no sólo filosóficas, y aumenta las posibilidades de alcanzar la verdad. De hecho, el llamado «sesgo de confirmación» se aplica a la recopilación y búsqueda de razones, no a la evaluación ${ }^{22}$. Es un sesgo para confirmar cualquier tesis que uno esté defendiendo; por eso el objetivo es encontrar razones a favor de las propias ideas y en contra de los puntos de vista a los que uno se opone (de ahí la expresión myside bias, que puede entenderse como una cierta propensión que los humanos solemos tener para favorecer ciertos puntos de vista que confirmen nuestras propias creencias sin tener la suficiente evidencia de que esas creencias son verdaderas). Pero argumentar exige tanto generar argumentos como evaluarlos y, cuando se trata de evaluarlos, las personas pueden aceptar los buenos argumentos de los demás siempre y cuando se encuentren en una disposición psicológica apropiada, i.e., en la disposición que les permita admitir buenas razones. Si éste es efectivamente el caso, incluso cuando una persona tiene una enorme confianza en su punto de vista, puede estar dispuesta a modificarlo si los argumentos del otro así lo sugieren. Esto mostraría que la argumentación (pero más propiamente la deliberación) evolucionó para funcionar en un entorno interactivo ${ }^{23}$, y el «sesgo de confirmación» se convierte en parte de una manera elegante y útil de dividir el trabajo cognitivo. En efecto, dicho sesgo hace que cada parte presente un caso relevante a favor de sus puntos de vista para luego evaluar juntos (como lados no combatientes) los argumentos, donde cada parte controla la calidad de las razones proporcionadas por el otro y adapta sus argumentos a las objeciones específicas que se plantean. Este tipo de argumentación, que ayuda a explicar el cuidado que tenemos por respaldar nuestras creencias pero que, a la vez, puede servir para evaluar con otro un problema, replica, mutatis mutandis, la idea platónica de cómo funciona la discusión dialógica y, por ende, la investigación conjunta. Este proceso interactivo de producción y evaluación de argumentos implica una división del trabajo cognitivo que de hecho convierte el sesgo de confirmación en una característica útil de la mente en lugar de un mero error.

He enfatizado más arriba la relevancia de la división del trabajo cognitivo; en particular, la visión socrático-platónica de que la investigación es una tarea compartida muestra, como he indicado, que tanto la producción como la evaluación de argumentos pueden ayudarnos a mejorar nuestro desempeño epistémico, entre otras cosas, porque el debate dialógico ayuda a revisar nuestras propias creencias. Un aspecto interesante de esto deriva, una vez más, de Sócrates, quien, aparentemente, no está dispuesto a aceptar el testimonio del oráculo de Delfos con respecto a su propia sabiduría; cuando en la Apología platónica el oráculo le dice a Querefonte que nadie es más sabio que Sócrates

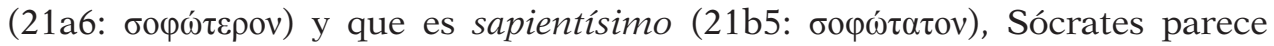
tener dudas. Sin embargo, los dioses no mienten ni se equivocan; Sócrates no es escéptico sobre los dichos del dios, sino que está procurando comprender el

22 Ver Mercier, H. y Sperberg, D., op. cit., pp. 218-228.

23 Mercier, H. y Sperberg, D., op. cit. pp. 224-227; 264-265. 
significado de esos dichos (probablemente, porque tiene una convicción firme acerca de su propia ignorancia $)^{24}$ : cree que lo que dice el dios es cierto, aunque aún no lo comprende ${ }^{25}$. Es más, Sócrates entiende la declaración de Apolo

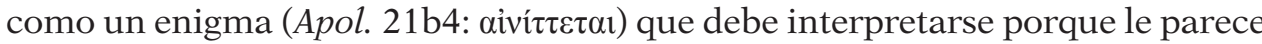
que los demás son más sabios (o más competentes) que él. Sin duda, tiene una convicción firme (es decir, una creencia basada en lo que considera que son buenas razones) respecto del hecho de que es ignorante. Si esto es así, no niega la autoridad de lo que dice el dios acerca de él, pero no parece entender lo que dice. Entonces, como al mismo tiempo está convencido de que lo que dice el dios no puede ser una trivialidad o una falsedad, procede a investigar el significado de lo que dice Apolo (a través del oráculo).

Finalmente, después de interrogar a los supuestos expertos (políticos,

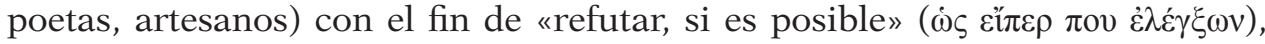
es decir, de "examinar» el apotegma del dios («Sócrates es sapientísimo»), Sócrates advierte que no entiende por qué es «sapientísimo», como dice el dios: lo que sucede es que ha sido capaz de reconciliar lo que dice el dios con lo que cree acerca de sí mismo (que es ignorante) mediante una interpretación de la sentencia délfica: «soy el más sabio porque no creo saber lo que no sé». Dicho de otra manera, Sócrates no sufre de una ilusión de conocimiento; es consciente de los límites de su propio conocimiento. Cuando comienza a poner en práctica su método para examinar si realmente hay alguien que dice ser un experto en un campo de especialización dado, comienza con el político (Apol. 21c3). Al recordar su encuentro con el político, enfatiza el hecho de que se dirigió a «uno de los que parecían ser sabios» (probablemente, en el sentido de «uno de los que fue considerado sabio o competente» o "que tenía una reputación como

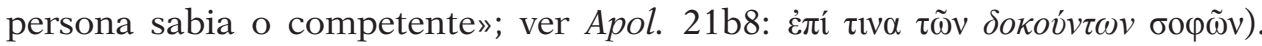
Después de examinarlo (es decir, después de poner en práctica el método de examen que consiste en preguntas relacionadas con el supuesto conocimiento que el supuesto experto dice poseer), Sócrates concluye que es más sabio que el político porque, aunque ninguno de ellos sabe nada que merezca la pena, el político cree que sabe algo, aunque en realidad no lo sabe. Como recuerda Sócrates, al conversar con el político (21c5) le parecía que no sólo muchas otras personas creían que el político era sabio, sino especialmente el propio político (esto recuerda el efecto Kruger-Dunning: cuanto más ignorante y, en general, incompetente es la persona, se siente más segura de que no es ignorante o incompetente). Sin duda, este énfasis no es trivial (el supuesto experto pensó que era un experto, pero tal vez no tenía suficientes razones para creerlo). De hecho, el político, un supuesto experto, muestra el estado psicológico del individuo que no tiene dudas sobre su propio conocimiento y, no obstante,

24 Cfr. Platón, Apol. 21b7, y la discusión de Brickhouse, T. C. y Smith, N.D., The Philosophy of Socrates, Westview Press, Boulder, Colorado: 2000, p. 77.

25 Sócrates dice que va a investigar lo que el oráculo ha dicho sobre él «con gran

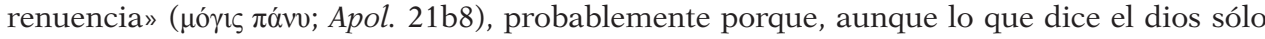
puede ser verdad, él cree que es ignorante. 
no puede responder satisfactoriamente a las preguntas de Sócrates, revelando así una cierta arrogancia epistémica ${ }^{26}$. Sócrates, por otro lado, no sabe y tampoco cree saber (Apol. 21d4-7), por lo que concluye que es más sabio que

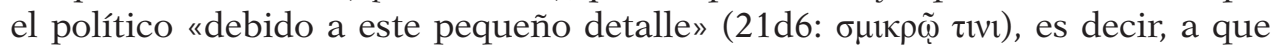
no cree saber lo que no sabe. En Apol. 21b-d la «conciencia» de la ignorancia que Sócrates afirma poseer se opone más tarde a la Ignorancia Obstinada (IO) que menciona nuevamente en Apol. 29b1-2 («la ignorancia más culpable»

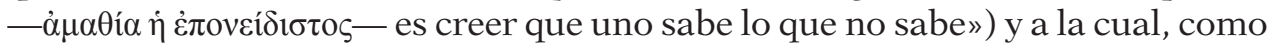
veremos, vuelve con un énfasis peculiar en el Sofista, un diálogo seguramente muy tardío en la producción filosófica de Platón, pero que mantiene intacta una idea decisiva de un diálogo temprano como la Apología de Sócrates.

Esta última parte de mi discusión puede leerse como una presentación bien conocida de algo que todos ya sabemos sobre el extravagante Sócrates: que es

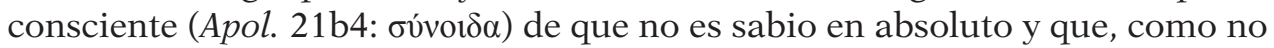

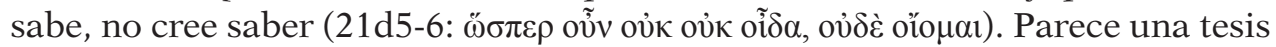
muy simple, pero no hay duda de que Platón no la consideró banal; además, pensó que era el $a b c$ de la tarea filosófica. En el Sofista la repite de nuevo y con un énfasis un poco más dramático, cuando dice que saber en sentido estricto es creer que uno sabe únicamente lo que sabe (

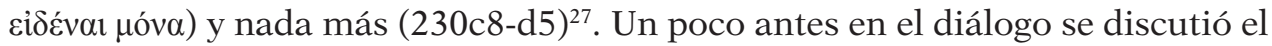
tema de la ignorancia como una cierta «deficiencia en el alma»; el Extranjero

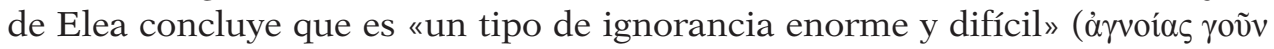

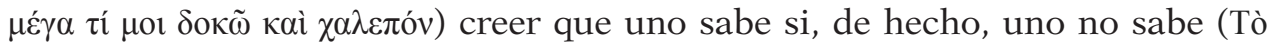
$\mu \eta ̀ ~ \kappa \alpha \tau \varepsilon ı \delta ́ ́ \tau \alpha ~ \tau \imath ~ \delta о к \varepsilon i ̃ v ~ \varepsilon i ́ \delta \varepsilon ́ v \alpha l ; ~ S o f i s t a ~ 229 c 1-5)^{28}$. Parece entonces que la IO, no la IS, fomenta una arrogancia epistémica que impide que una persona pueda comprender adecuadamente un argumento o razón mejor. El que está en ese

${ }^{26}$ Aunque el texto no dice que el político no fue capaz de responder a las preguntas de Sócrates, seguramente deberíamos suponerlo; esto es así porque Sócrates dice que, después de su examen, se convirtió en objeto de odio del político y de muchos de los que estaban presentes, quienes habían supuesto (y probablemente aún suponían) que el político era un verdadero experto en su área de especialización (Apol. 21c-d). Lo que hizo Sócrates fue arruinar la reputación del político, al mostrar que no era capaz de justificar su supuesto conocimiento y que, por ende, no era un verdadero experto.

27 En Gorgias 458a6 la refutación es «un bien mayor» ( $\mu \varepsilon i \check{\zeta} o v$ ả $\gamma \alpha \theta$ óv) y ahora en el Sofista se pone el énfasis en el hecho de que el alma misma no puede liberarse de aquello que le impide adquirir el conocimiento hasta que el que refuta avergüence al refutado. Tal vez esto es parte de la manera «escandalosa» con la que Sócrates pretende despertar a la persona para que no crea que sabe lo que, en realidad, no sabe. Pero la refutación como tal tiene un carácter terapéutico en la medida en que permite al individuo deshacerse de la ignorancia, que es una forma de maldad.

28 En Menón 84a Platón muestra los dos momentos de la conversación filosófica y el estado psicológico de quien es interrogado: antes de la conversación el esclavo no sabía lo que era una superficie de ocho pies («todavía no lo sabe», recuerda Sócrates en 84a5-6: ov̉ò̀

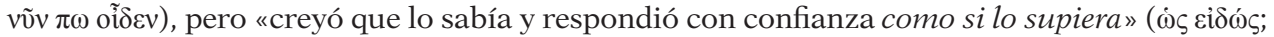
84a6-7). Después del debate filosófico, el esclavo está en aporía, está perplejo, y aunque no sabe, no cree que sabe. 
estado psicológico no es capaz de dudar de sí mismo: ningún argumento es persuasivo en contra de la IO de alguien.

Como es obvio, se trata de un estado de ignorancia radicalmente opuesto a la IS. En un notable pasaje de Teeteto 176e-177a se muestra que la obcecación o terquedad (que es equivalente a la estupidez) implicada por Platón (estupidez que podría asociarse a la ignorancia en otras partes del diálogo; véase Teeteto176c) no se puede entender en el sentido de falta de ciertos contenidos cognitivos, sino que es un estado psicológico consistente en ser incapaz de admitir el propio error y creer que uno sabe lo que no sabe. Lo que este tipo de actitud describe es la ausencia de humildad intelectual, que es la que ayuda a evitar la ilusión del conocimiento o, una vez que se tiene esa ilusión, a neutralizarla. En realidad, se trata de una sugerencia platónica que, mutatis mutandis, también está presente en un diálogo temprano (o de transición) como el Gorgias: si uno ha aceptado la refutación del propio logos a conciencia (lo que significa asumir las inconsistencias que se siguen de los supuestos iniciales), uno podrá transformar su creencia en la dirección correcta, ya que será capaz de advertir la fragilidad de su supuesto conocimiento ${ }^{29}$. Ese tipo de actitud se puede ver en el ilustrativo y conocido pasaje del Gorgias de Platón que ya he mencionado al pasar (458a2-b1), donde Sócrates declara que le agrada ser refutado si está diciendo algo falso, pero que no está más descontento de ser refutado que de

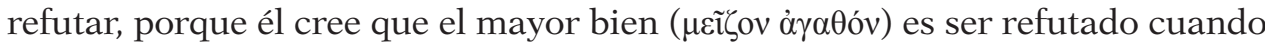
uno dice una falsedad, ya que no existe un mal tan grande para alguien como una opinión o creencia falsa ( $\delta$ ó $\alpha \alpha \varepsilon \varepsilon \delta \delta \eta ́$ ). Cuando uno es refutado se deshace de un mal, es decir, de la IO, representada en el pasaje por la persistencia en tratar de mantenerse en un estado de ignorancia por no ser capaz de reconocer el propio error. Que Sócrates se alegra de ser refutado muestra la humildad que es necesaria para avanzar en la búsqueda de la verdad; que se complace en refutar a alguien más muestra una autonomía por parte de Sócrates, pero esa autonomía no debe entenderse en el sentido de que su propia opinión es incuestionable. Cualquier creencia es potencialmente refutable, pero si uno tiene buenas razones para creer lo que cree, uno puede permitirse corregir las creencias de otra persona ${ }^{30}$.

Sócrates cree que es ignorante (Apol. 21b4-5; 22c9-d1); los que están a su alrededor, en cambio, se perciben más sabios o competentes de lo que realmente son. En Apol. 21c, Sócrates afirma que los poetas, dados los conocimientos que poseen, piensan que también son muy sabios o competentes en otros dominios, lo cual es falso: la posesión de conocimiento en un cierto campo

29 Que la ignorancia de la que habla Platón en estos pasajes no puede ser ignorancia en el sentido de ausencia de ciertos contenidos cognitivos puede verse en República 585b3-4,

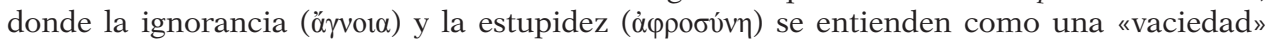

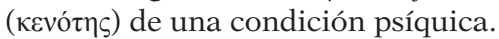

30 El vigor de esas creencias se muestra en el examen dialógico, es decir, si son capaces de resistir ese examen, hay una razón para pensar que son poderosas y que pueden seguir siendo confiables. 
de especialización no nos habilita a ser conocedores de otros contenidos cognitivos. La ventaja de Sócrates sobre los poetas es que no cree saber, cuando no sabe (es decir, no sufre una ilusión de conocimiento). Además, como se ha visto, algunos (como el personaje Filebo - en el pasaje antes citado de Filebo 12a7-8-, Trasímaco en República I y Calicles en la sección final de Gorgias 481b-505d) podrían no estar dispuestos a cambiar de opinión (aunque por lo general exigen que otros lo hagan). Esto puede sugerir que Sócrates era consciente del hecho de que las personas están menos abiertas a las creencias de los demás que pueden estar en conflicto con sus propias creencias. Si para Sócrates tanto la función del diálogo como la «investigación compartida» son tan importantes para avanzar en la búsqueda del conocimiento, debe haber sido consciente de nuestros propios límites para adquirir conocimiento. De hecho, la estructura epistémica de la sociedad (tanto de la sociedad que describe Platón como de la nuestra) abarca tales limitaciones y nos ofrece una manera de superarla mediante la división del trabajo cognitivo. Y Sócrates, como se sugirió anteriormente, era consciente de los beneficios de explotar tales divisiones del trabajo. Sin embargo, para aprovechar esos beneficios, se requiere un carácter intelectual particular; tal carácter no sólo requiere que el sujeto sea intelectualmente humilde, sino también intelectualmente autónomo. La autonomía no es una cuestión de pura independencia, sino de lo que se hace con la propia dependencia; de hecho, implica algún tipo de "gestión» de la dependencia. En cuanto a la virtud intelectual de la autonomía epistémica, reduce la pura dependencia epistémica de los demás y promueve una disposición a pensar críticamente para uno mismo al juzgar puntos de vista (como lo hace Sócrates), sin caer en un hiper-individualismo.

Así, la virtud de la autonomía epistémica también requiere que el sujeto sea capaz de identificar fuentes confiables de conocimiento (incluyendo a los expertos) y contenidos plausibles (como hace Sócrates) cuando tiene que depender epistémicamente de otros. Este monitoreo epistémico es, para decirlo de otro modo, parte de las habilidades que requerimos para poder explotar con éxito la división del trabajo epistémico.

\section{Las Razones de Sócrates PaRa PREgUntar y nO HACER AFIRMACIONES POSITIVAS y EL VALOR DE LA ACTITUD SOCRÁTICA EN CONTEXTOS POLÍTICO-DELIBERATIVOS}

Sócrates cuestiona las creencias todo el tiempo, profesa su ignorancia (Cármides 165b) y declara que pregunta porque no sabe y que su único propósito en el interrogatorio es "examinarse a sí mismo» (Cármides 166d-e; Laques 186d-e). Además, el diálogo (entendido como un debate argumentativo) es la investigación compartida y supone el abandono de un enfoque solipsista en la búsqueda de conocimiento. Pero para que el diálogo sea efectivo como una actividad compartida, debe ser una conversación sin rivalidad, sin «amor

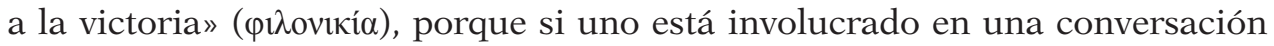


filosófica (concebida socrático-platónicamente), no está compitiendo o dando batalla para poder prevalecer sobre el otro interlocutor. Los hablantes verdaderamente filosóficos son aliados en vista de lo más verdadero ${ }^{31}$, pues necesitan explotar la división del trabajo cognitivo que ya existe y para la cual requieren al menos las dos virtudes intelectuales mencionadas (humildad y autonomía). Es importante dejar claro que nada de lo anterior se limita a creencias u opiniones filosóficas. En todos los dominios de la vida (dada nuestra necesidad de verdad y nuestras limitaciones) estamos comprometidos a ser capaces de explotar las divisiones de trabajo epistémico en el sentido antes indicado.

Tanto para «consumir» como para producir conocimiento dependemos de los demás. De este modo, la regulación apropiada de nuestras creencias va de la mano de ciertas actitudes y disposiciones que posee el sujeto intelectualmente humilde y autónomo. Esta regulación es muy importante en todos los aspectos de la vida, pero para nosotros, como miembros de sociedades democráticasrepublicanas (o que pretenden serlo), es sumamente importante en lo que respecta a nuestras creencias políticas. La democracia republicana requiere que sus ciudadanos sean al menos mínimamente competentes; para serlo, deben estar al menos bien informados o ser capaces de formar opiniones precisas sobre una variedad de problemas (económicos, educativos, ambientales, migratorios, etc.). Y, como cabría esperar dado lo anterior, el debate político entre ciudadanos que explota la división del trabajo epistémico y cognitivo no debería mostrar la polarización y la desinformación que hoy en día estamos acostumbrados a ver ${ }^{32}$. Los participantes en este tipo de discusiones terminan con una mejor información, con posiciones más articuladas y con una comprensión más profunda (y por tanto crítica, en el sentido de escrutadora) del punto de vista de otras personas. Es importante destacar que las opiniones de quienes participan de los debates tienden a converger a un compromiso epistémicamente razonable e involucran un cambio de opinión de, al menos, algunas de las partes.

Como es esperable, la deliberación entre los ciudadanos — que ejemplifica la humildad intelectual y la autonomía- puede funcionar o, de hecho, funciona (al menos a veces). Y dado que estas virtudes intelectuales parecen ser esenciales para una vida cívica saludable, deberíamos enfocarnos en el desarrollo de los caracteres intelectuales de los ciudadanos para ejemplificar las actitudes y disposiciones relevantes. Si es cierto que para consumir y para producir conocimiento dependemos de los demás, deberíamos ser exigentes con nuestros conciudadanos, esto es, deberíamos exigirles que se informen y se

31 Platón, Filebo 14b1-7. La misma idea aparece en un diálogo temprano (Laques 194a).

32 La clave para neutralizar la polarización (que implícitamente sostiene que el adversario político no sólo está completamente equivocado, sino que sus ideas políticas son repugnantes) hay que encontrarla en la manera de hacer que las personas piensen más allá de sus propios intereses, y de que sientan la necesidad de examinar sus creencias (sobre este punto cfr. Sloman-FernBach, op. cit., p. 260-261). 
formen apropiadamente, de manera que a la hora de elegir a sus autoridades lo hagan evaluando de modo apropiado no sólo los pros y contras de cada posición, sino también las posibilidades que las posiciones que tengan mayor chances de lograr obtener representación en el gobierno sean capaces de lograr consensos racionales, donde las partes en pugna deben ser capaces de ceder en algo.

Si tuviéramos éxito en la enseñanza de esas actitudes y disposiciones, lograríamos hacer que nuestros conciudadanos trabajaran epistémicamente de modo correcto con los demás y, de esa manera, se podría comenzar a abordar las preocupaciones competitivas de la tradición antidemocrática que (correcta pero irónicamente) comienza con la República de Platón.

\section{BibLIOGRAFÍA}

Brickhouse, T. C. y Smith, N. D. (2000). The Philosophy of Socrates. Boulder, Colorado: Westview Press.

Burnet, J. (1976). Platonis Opera (Recognovit brevique admnotatione critica instruxit Ioannes Burnet), Tomvs I-IV. Oxford: Oxford Classical Texts (reprinted).

Coady, C. A. (2012). What to Believe Now: applying epistemology to contemporary issues. Chichester: Wiley-Blackwell.

Davidson, D. (2005). «Dialectic and Dialogue», en: Davidson, D., Truth, Language, and History. Oxford: Oxford University Press, 251-259

Davidson, D. (2001). «First Person Authority», en: Davidson, D., Subjective, Intersubjective, Objective. Oxford: Clarendon Press, 3-14.

Dorion, L.-A. (2011). Que sais-je? Socrate. Paris: Presses Universitaires de France.

Gadamer, H.-G. (1999). Die Idee des Guten zwischen Plato und Aristoteles, en: Gadamer, H.-G., Gesammelte Werke 7. Griechische Philosophie III. Tübingen: J. C. B. Mohr (Paul Siebeck), 128-227.

Giannantoni, G. (2005). Dialogo socratico e nascita della dialettica nella filosofía di Platone (Edizione postuma a cura di Bruno Centrone). Napoli: Bibliopolis.

Gill, C. (2004). «Afterword: Dialectic and the Dialogue Form in Late Plato», en: GILL, C. y McCabe, M. M. (eds.), Form and Argument in Late Plato. Oxford: Clarendon Press, 283-311.

Gill, C. (2007). «Form and outcome of arguments in Plato's Gorgias», en: Erler, M. y Brisson, L. (eds.) Gorgias-Menon. Selected papers from the Seventh Symposium Platonicum. Sankt Augustin: Academia Verlag, 62-65.

Goldman, A. (2001). «Experts: Which Ones Should You Trust?», en: Philosophy and Phenomenological Research vol. 63, №1, 85-106.

Goldman, A. y Olsson, E. J., «Reliabilism and the Value of Knowledge», en: Haddock, A., Millar, A. y Pritchard, D. (eds.) Epistemic Value. Oxford: Oxford University Press, 19-41.

Kahn, C. (1996). Plato and the Socratic Dialogue. The Philosophical Use of a Literary Form. Cambridge: Cambridge University Press.

Kruger, J. y Dunning, D. (2009). «Unskilled and Unaware of It: How Difficulties in Recognizing One's Own Incompetence Lead to Inflated Self-Assessment» en: Psychology, 1, 30-46

Le Morvan, P. y Peels, R. (2016). «The Nature of Ignorance: Two Views», en: Peels, R. y Blaauw, M. (eds.), The Epistemic Dimensions of Ignorance, Cambridge: UCambridge University Press, 12-32 
MacPartland, K., «Socratic Ignorance and Types of Knowledge», en: Bussanich, J. y Smith, N. D. (eds.), The Bloomsbury Companion to Socrates. London-New Delhi-New York-Sydney: Bloomsbury, 2013, 94-135.

Mercier, H. y Sperberg, D. (2017). The Enigma of Reason. Cambridge, Massachusetts: Harvard University Press.

Natali, C. (2007). «Due dissertazioni scritte in fretta. Gadamer e Davidson sul Filebo di Platone»,en: Méthexis XX, 113-143.

Peels, R. y Blaauw, M. (2016). «Introduction», en: Peels, R., Blaauw, M. (eds.) The Epistemic Dimensions of Ignorance. Cambridge: Cambridge University Press, 1-11.

Pritchard, D. (2016).«Ignorance and Epistemic Value», en: Peels, R. y Blaauw, M. (eds.) The Epistemic Dimensions of Ignorance. Cambridge: Cambridge University Press, 132-143.

Renaud, F. (2008). «Les Mémorables de Xénophon et le Gorgias de Platon : étude comparative», en: Narcy, M. y Tordesillas, A. (eds.) Xénophon et Socrates. Paris: Vrin, 161180.

Sloman, S., Fernbach, P. (2017). The Knowledge Illusion: Why we never think alone. London: Macmillan.

Taylor, C. C. W. (2008). «Socrates», en: Taylor, C. C. W., Pleasure, Mind, and Soul. Selected Papers in Ancient Philosophy. Oxford: Clarendon Press,.

Vallejo Campos, A.,(2017). "Sócrates» en: Vallejo Campos, A. y Vigo A. G., Filósofos griegos: de los sofistas a Aristóteles. Pamplona: Eunsa, 93-104.

Vigo, A. G. (2018). Platón, Apología de Sócrates. Buenos Aires: Ed. Colihue.

Waterfield, R. (2013). «The Quest for the Historical Socrates», en: Bussanich, J. y Smith, N. D. (eds.), The Bloomsbury Companion to Socrates. London-New Delhi-New YorkSydney: Bloomsbury, 1-19.

Zagzebski, L. (2012). Epistemic Authority: A Theory of Trust, Authority, and Autonomy in Belief. New York: Oxford University Press.

Zimmerman, M. J. (2008). Living with Uncertainty. The Moral Significance of Ignorance. Cambridge: Cambridge University Press.

Pontificia Universidad Católica de Chile

MARCElo D. BOERI mboeric@uc.cl

[Artículo aprobado para publicación en febrero de 2021] 